\title{
Game Playing: Proposing and Developing Queen Challenge Puzzle Game from 1 to 25 Levels
}

\author{
Hussain Mohammad Abu-Dalbouh ${ }^{1}$, Fahad Almansour ${ }^{2} \&$ Nehal Aldowighri ${ }^{3}$ \\ ${ }^{1}$ Department of Computer Science, College of Science and Arts, Qassim University, Unaizah, Saudi Arabia \\ ${ }^{2}$ Department of Computer Science, College of Science and Arts, Qassim University, Ar Rass, Saudi Arabia \\ ${ }^{3}$ Department of Computer Science, College of Computer, Mustaqbal University, Unaizah, Saudi Arabia \\ Correspondence: Hussain Mohammad Abu-Dalbouh, Department of Computer Science, College of Science and \\ Arts, Qassim University, Unaizah, Saudi Arabia.
}

Received: March 15, 2021

Accepted: April 6, $2021 \quad$ Online Published: April 13, 2021

doi:10.5539/cis.v14n2p87

URL: https://doi.org/10.5539/cis.v14n2p87

\begin{abstract}
In recent decades, massive improvements in graphic sophistication have begun to produce declining returns. The creative focus in game development has shifted to artificial intelligence. The queens' task game is part of a sequence of popular games. It is the challenge of putting $\mathrm{n}$ chess queens on a game board such that no two queens are threatening each other. The plan does not involve two queens sharing the same row, column or diagonal. Each column contains exactly one queen, each row contains exactly one queen, and each diagonal contains exactly one queen. For every level in the game, there are many ways to solve it. For example, there are 92 solutions to the $8 \times 8$ problem. There are many levels in the literature, but each level should be downloaded separately. Thus, it causes a lot of difficulties for players, and they should download each level to complete the challenge. This will lead to more time and effort being spent by the players, and the cost of each level will cost the players more and more. As a result, the number of players who want to play this game will decrease. The aim of this paper is to incorporate a number of levels in order to save time, money and effort by downloading each level separately. This paper also aims to develop the proposed prototype and display all the solutions while playing a puzzle game at any level. The proposed game was tested by a questionnaire-based empirical study. Descriptive statistics on the questions revealed that the players had achieved the objectives of the game by applying their skills and knowledge and that the players had positive emotions about the effectiveness of the proposed game.
\end{abstract}

Keywords: game, design, models, implementation, genetic algorithm, fun, challenge, motivation, usefulness

\section{Introduction}

Play is more than a simple physiological action or a mental reflex. It goes beyond the limits of physical or simply natural action. Play contains something that goes beyond the prompt needs of life and gives importance to the activity (Johan and Homo, 1955). A very valuable approach to see scientific puzzles is like a game. Puzzle is like a game that people play against themselves. Game Hypothesis is a well-created part of science that focuses on portraying how different entertainers interact with each other under certain circumstances.

In recent years, a lot of people have access to computers on a wide scale, and a lot of them are playing games. Games can develop the cognitive abilities. Games require abstract, intensive skills to win, skills that classroom cannot educate students. Computer game users have a desire for not only games in general, but also for quality games that provide them with entertainment. Games need to be created in order that is well considered out and well organized to provide the user with the best possible experience. The $\mathrm{N}$-queens puzzle is well known to computer researchers. It is a big puzzle speculation documented as 8-queens puzzled. The first is credited to the chess player Max Bezzel and involves placing eight queens on an $8 \times 8$ chessboard in such a way that none of them can find another by using the standard improvement of the chess queens. That is, on each line, column and diagonal line, only one queen can be set. The 8-queens puzzle was later summed up as an N-queens puzzle, with similar standards but putting $\mathrm{N}$-queens on the $\mathrm{N} \times \mathrm{N}$ board (Arnoldy, 2006).

The game has many levels from $1 \times 1$ to $25 \times 25$, but each level can be found in a separate game. This is going to create many obstacles for the players. They should download each level to complete the game, but they will 
spend more time and effort and the cost of each level will be costly. Thus, this study will reduce the number of players who play the game. This project will collect all levels and solutions for each level in one program to help players, save time and effort and lowering the cost to players. In the game world, there are no more games to test the intelligence of players, but this prototype program will improve the intelligence of players from 13 years of age and older.

The aim of this study is to present solutions to the problem of the $n$ queens on the $n \times n$ board. In fact, a simple random algorithm based on a strategy of trial and error should be used to calculate solutions. It should be taken into account that the user could provide the initial conditions for a solution, i.e. that the user could place a certain number of queens and ask the program to look for a possible solution. In addition, the user interface will allow a screen display solution, highlighting the queens that initially placed by the user and generated by the program. The scope of the project is limited to Visual Studio. Net. The application input will be limited to simple inputs for the user's strategic move to solve the puzzle. The only output is the entertainment that is the result of the input.

\section{N-Queen Puzzle Game}

\subsection{Problem}

The queens puzzle challenge is part of a collection of famous puzzles. It is a matter of putting the chess queens on a chessboard, with the aim that no two queens are attack each other. The answer requires that no two queens share the same row, column, or diagonal. Each column contains exactly one queen, each row contains exactly one queen, and each diagonal contains exactly one queen.

There are a number of ways to solve each level. There are 92 solutions to the $8 \times 8$ problem, for example. The game has many levels from $1 \times 1$ to $25 \times 25$, but each level is found in a separate game, making it difficult for players. They should download each level to complete the game, therefore, players can spend more time and effort. In addition, the cost of each level will be high for the players. Consequently, the number of players who play this game will decrease.

\subsection{Purpose of the Study}

The purpose of this study is to gather all levels in a single game with a set of scenes, and it will have advantages in terms of the total cost of effort, time and money. In addition, this study will add additional levels to 25 to make the game more exciting and challenging even for brilliant minds. In addition, this game serves as a specific, mature gathering to evaluate their knowledge, learn basic leadership and perspective. A player will gradually start from the easiest to the hardest level, and the game will provide all possible solutions for all levels.

\subsection{Motivation and Study Questions}

Motivation is the mental quality that leads people to achieve an objective. Acing a language may be an objective for language learners. For other people, the informative capacity or even the essential relational capacity could be objective. The present society is knowledgeable in a variety of forms of excitement. Games talk to one of these structures and can cover a wide range of exercises or themes. In addition, computerized reasoning analysts have for quite a long time taken a shot at building game-playing frameworks that are suited to coordinating brains with the most grounded human beings in the world. Queens's problem, for example, is a mainstream game that has an extraordinary follow-up in many parts of the world. One of the most useful ways to play these games is through a computer. In addition, bringing the queens problem to the computer would make it possible for people to use it without buying a chessboard and pieces.

Despite this, the queens challenge puzzle has many levels from $1 \times 1$ to $25 \times 25$ but each level is found in a separate game and significant queries that continue in the minds of users remains unanswered. For example, Should each level be downloaded to complete the game separately? How do you incorporate several levels from $1 \times 1$ to $25 \times 25$ of the puzzle game? How will the proposed puzzle game prototype implement? What factors motivate players to download the queen's challenge puzzle? Is it possible to show all the solutions while the player plays the puzzle game at any level?

\section{Related Works}

Games are known also as computer games. They are played on a desktop with computer interface gadgets, such as a mouse or a joystick or gamepad. Video is running by the player on the computer screen, and the sound is transmitted by microphones or by earplugs. Computer is a systematic drawing of individuals around the world. With computer games, it is steadily rising as one of the adaptable games, offering unparalleled help to computer games. Computer game picks up ubiquity mainly in view of its top-notch preparation power. Computer games are 
programs that empower a player to work together with a virtual game condition for fun and entertainment. Numerous types of computer games are available, ranging from conventional games to more advanced computer games, such as simulation games and experience games. Puzzles and logic games are a lot of smart entertainment that makes them my favorites. Puzzles could be played for a considerable length of time, without getting exhausted by rehashing the same activity, additionally part of the rationale games has arbitrary level generator (like Bubble Breakers) or are randomized in one way or another, then you do not play the same level over and over. They hone your psyche and train tolerance, which is of value to all. Machine learning and its methods can be used to analyze emotions (Bikku and Sree, 2020). Artificial have become very popular in many fields (AL-Obaidi et al., 2020). The lecturer's major concern is the difficulty of teaching algorithm and programming course (Nasa et al., 2019).

Sudoku is a game based on logic, not a game based on arithmetic. Sudoku is an easy-to-learn puzzle for logical number placement (Boyer, 2009). "For Su-ji wa dokushin ni kagiru, the term Sudoku is limited, which means "the numbers must be single". 81 cells are involved in a Sudoku ambiguity, grouped into nine parts, lines and districts. The task is to place the numbers from 1 to 9 directly in the empty cells therefore, in each row, section and $3 \times 3$ district, each number appears only once.

The 15-puzzle is a sliding problem that remembers the edge of the numbered square tiles for an unusual request with one tile missing. Moreover, the conundrum exists in different sizes, especially the more modest 8 -puzzle. On the other hand, if the size is $3 \times 3$ tiles, the conundrum is known as 8 -confound or 9-perplex, and if $4 \times 4$ tiles, the enigma is known as 15-bewilder or 16-astound named separately for the number of tiles and the number of spaces.

Word Search, Word Find, Secret Word Perplex is a word game that includes letters of words placed in a structure that generally has a rectangular or square shape. The aim of this puzzle is to discover and check all the words inside the holder. Words may be determined on a level plane, vertically or diagonally. Much of the time a summary of the hidden words is given, even more testing puzzles may allow the player to understand them. Many word search confounds have a subject to which all hidden words are associated.

Crossword is a word puzzle that normally appears as a square or a rectangular matrix of white and dark shaded squares. The aim is to fill white squares with letters, molding words or articulations, by clarifying snippets of data that lead to appropriate reactions. In dialects that are left-to-right, the fitting reactions of words and joints are placed in the structure from left to right and through and through. Hidden squares are used to isolate words or joints.

\section{Implementation}

The context diagram is a description of the developmental that demonstrates the implementation limits, the outside elements that link to the approach, and the significant data streams between the entities and the system. Figures 1, 2 and 3 show the context, Level-0 and level 1 diagrams. At a high level of information, the level-0 diagram describes a system of major processes, data flows, and data stores. The Level-1 diagram illustrates the processes consisting of a single process with a Level- 0 diagram and how data travels from and through each of these processes. For all Level-0 processes, level-1 diagrams may not be needed (Hoffer, 2011). 


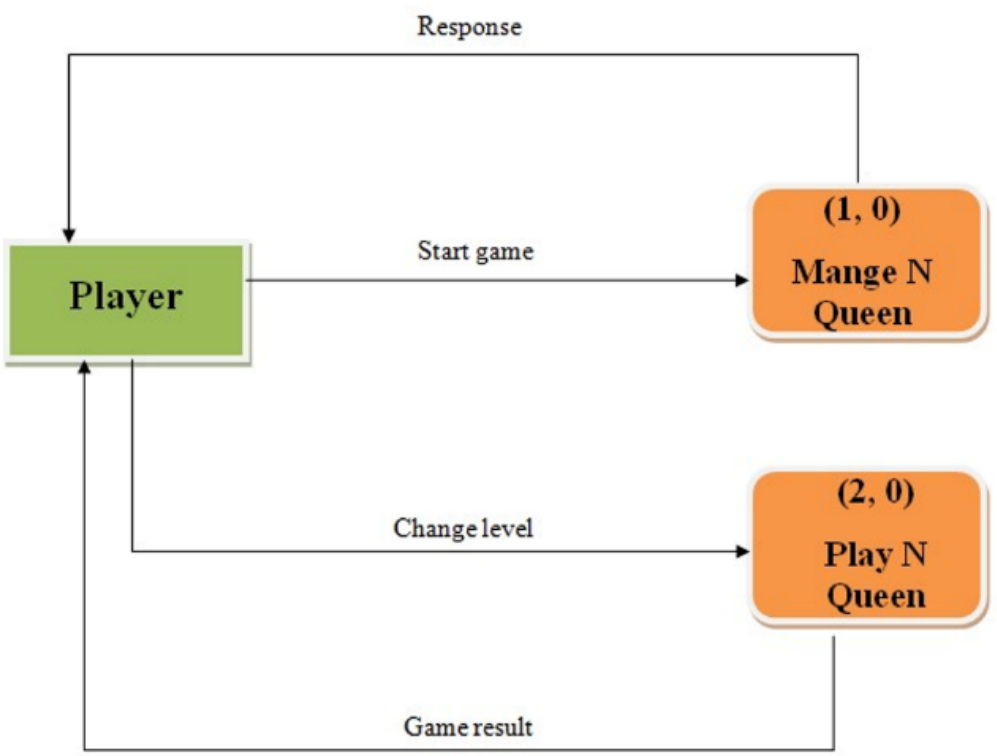

Figure 1. Context Diagram

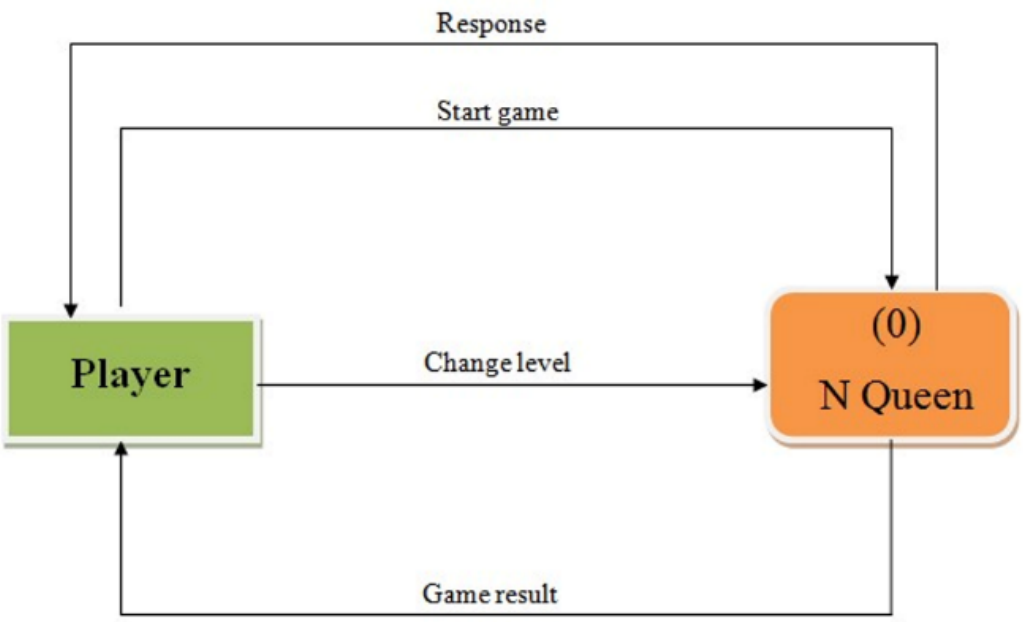

Figure 2. Level-0 Diagram 


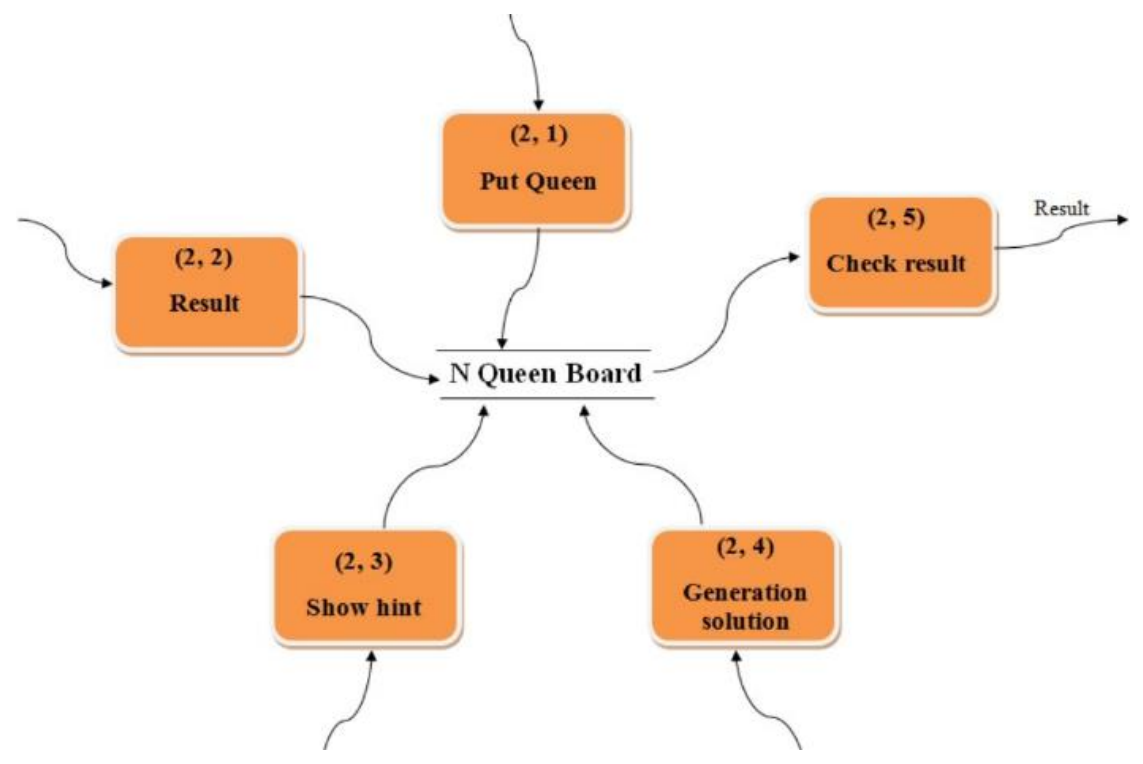

Figure 3. Level-1 Diagram

\section{- Splash screen 15 crossword}

This is the first screen shown to the player when the application is launched as shown in Figure. 4. The start screen is a user interface implemented as an 'Activity' in queen challenge game. Splash screen activity is will be started when the game is launched. It is responsible for waiting some time and launching a next activity in a new thread.

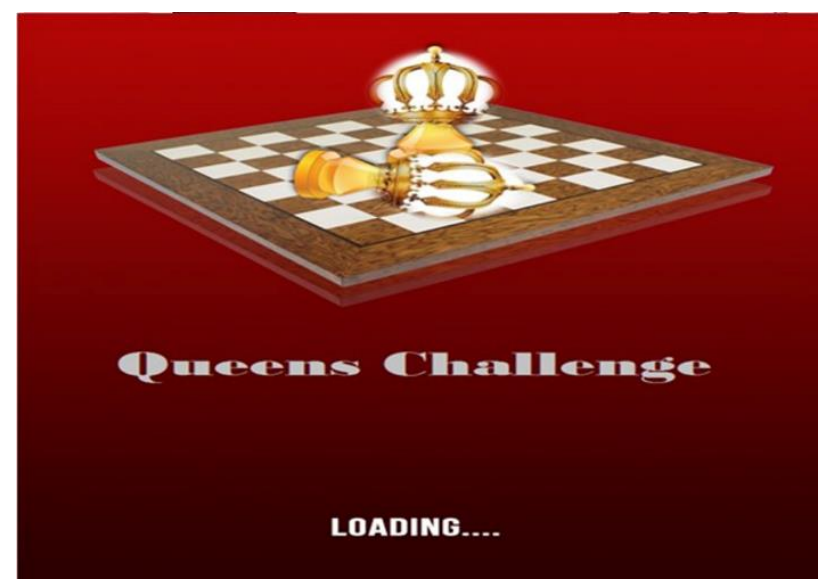

Figure 4. Splash Screen

\section{- Game Activity}

This main screen is launched after splash screen activity. The left side of the screen contains the basic choices of the application and the right side is a fixed frame of the stages of the game as shown in Figure 5. 


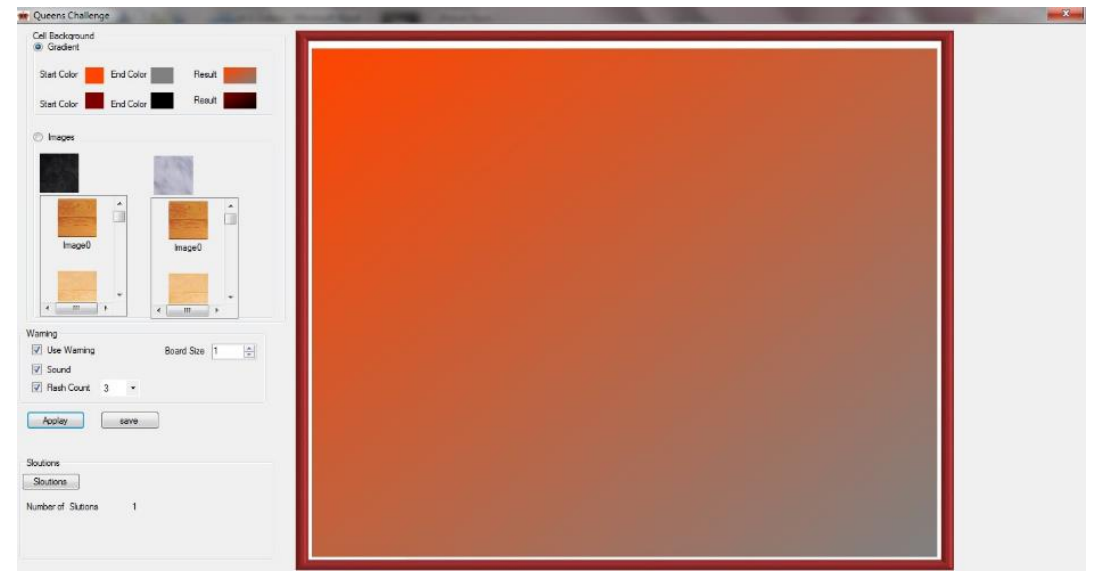

Figure 5. Game Activity

To change the cell background style or color, select color from gradient or select images from images then press the apply button to view the changes and pressed the save button to save the changes as shown in Figure 6. Warnings contain important choices for the application. Use warning to prevent the player to put queen in the wrong place. Board size on select any size from 1 to 25 the chessboard will be appear and the user is prompted to select any level to play. Sound it can be applause sound when the player win and a beep sound when the player put queen in the wrong place. Flash Count to it can be 1,2 or 3 . To view the changes press the apply button and to save the changes press the save button as shown in Figure 7.

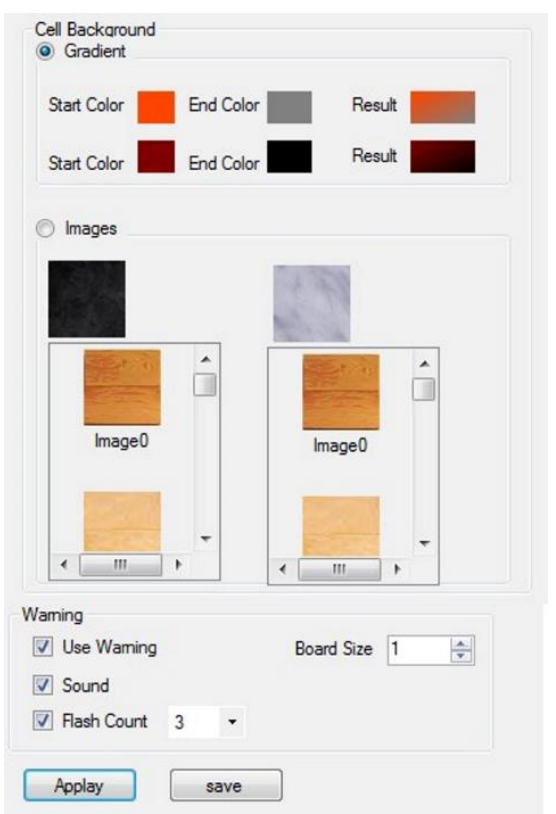

Figure 6. Game Activity - Change Cell Background/ Warning 


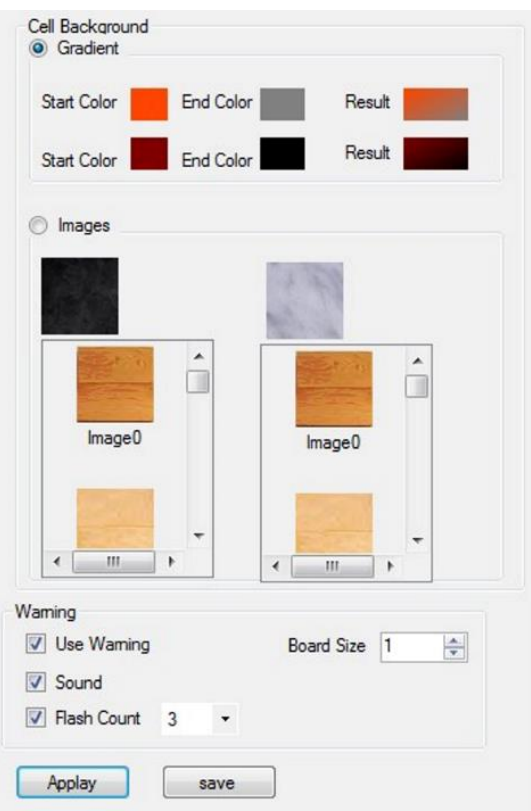

Figure 7. Game Activity - Warning

Figure 8 shows that the user select level four a chessboard $4 \times 4$ will be appears. The level of the game can save when the user leaves the current game screen by pressing save button. When the game is "Over" it displays a dialog box stating the game is won. Next screen captures depict that game is won. When the player finished the level will appear message notify him to go to the next level as shown in Figure 9.

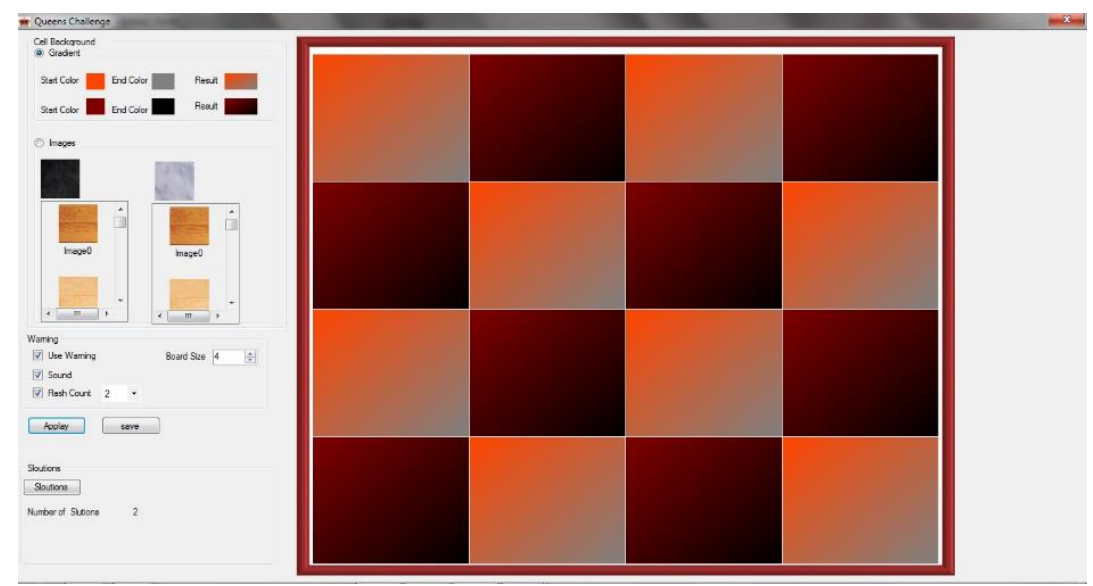

Figure 8. Game Activity- Choose Level 4 


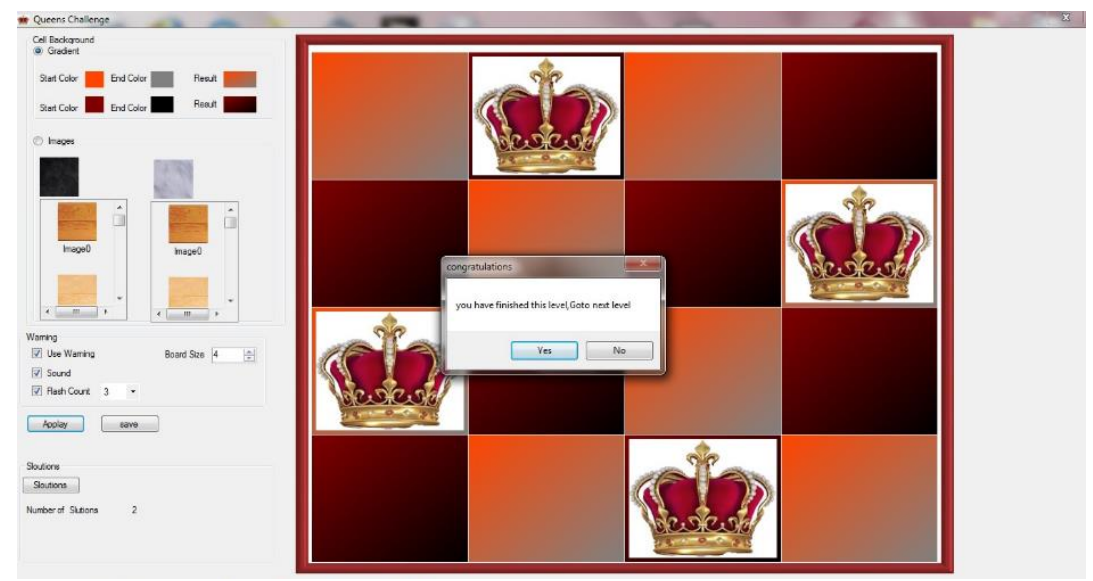

Figure 9. Game Activity

For any level, the player can know the possible numbers of solution. To view these solutions press solution button and move through right row. For example, level four has two solutions. Figures (10-14) are showing the choosing level and view solutions from the player.

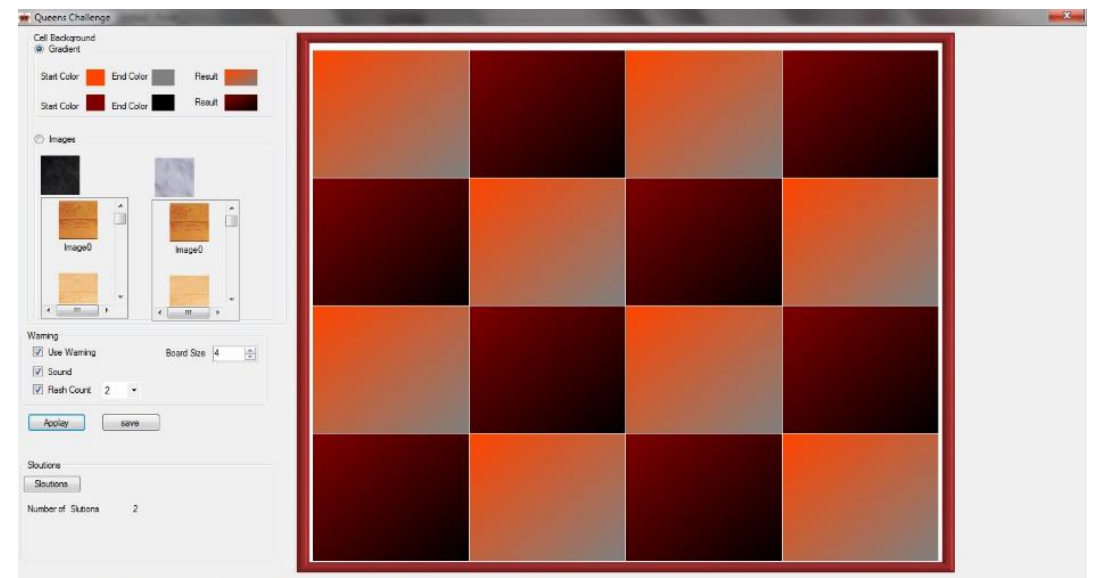

Figure 10. Game Activity -View Solution- Choose Level 4

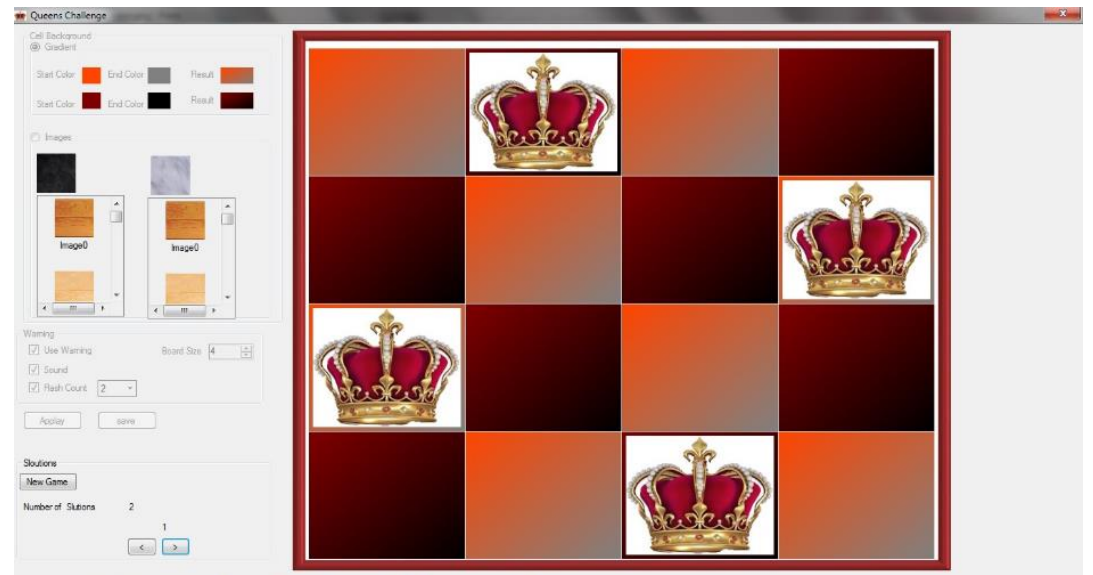

Figure 11. Game Activity -View solution- Choose Level 4 


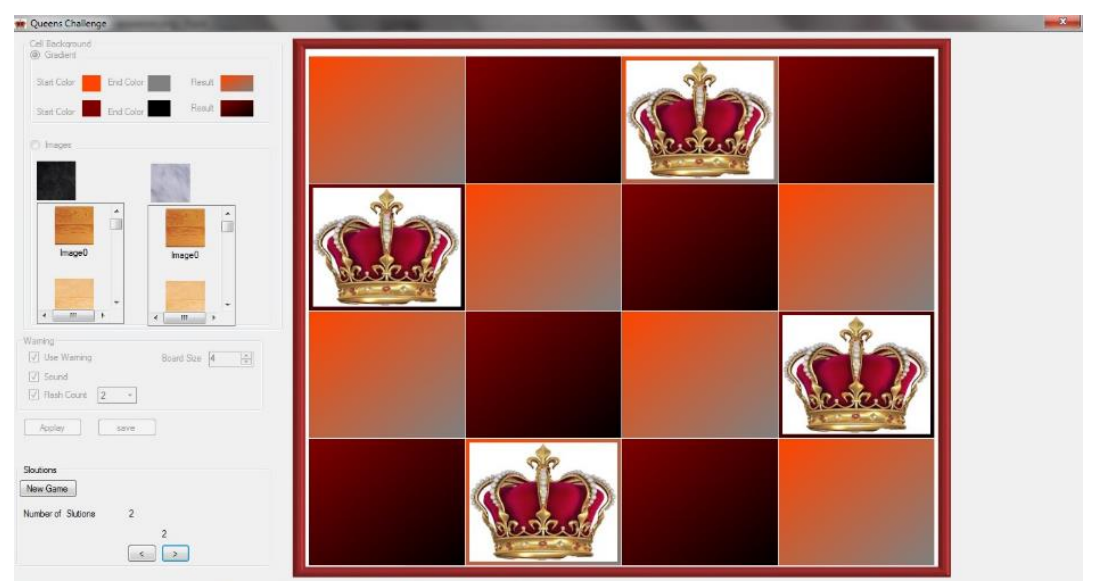

Figure 12. Game Activity -View solution- Choose Level 4

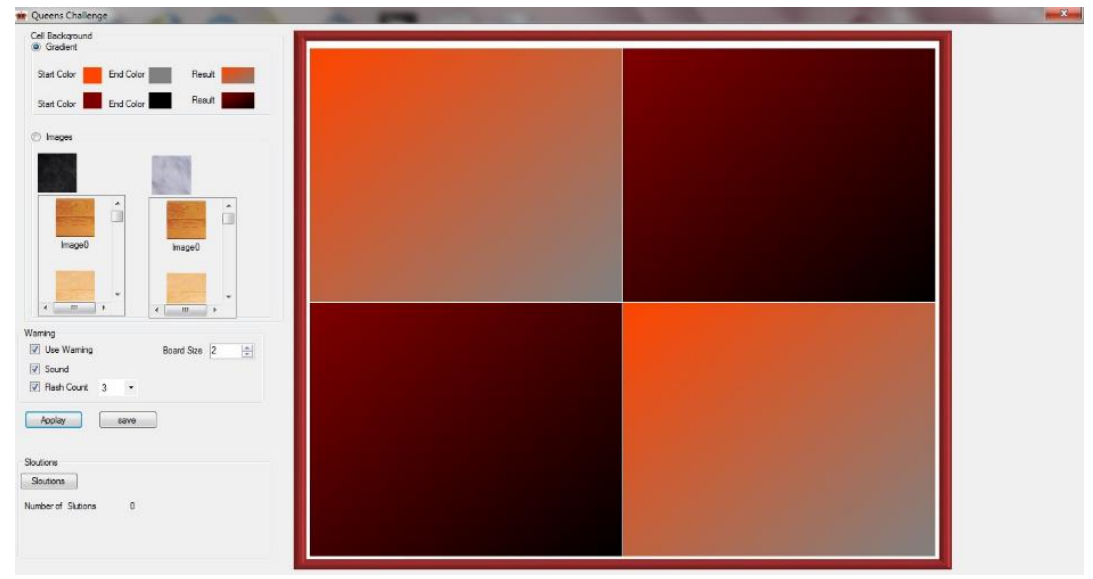

Figure 13. Game Activity- Choose Level 2

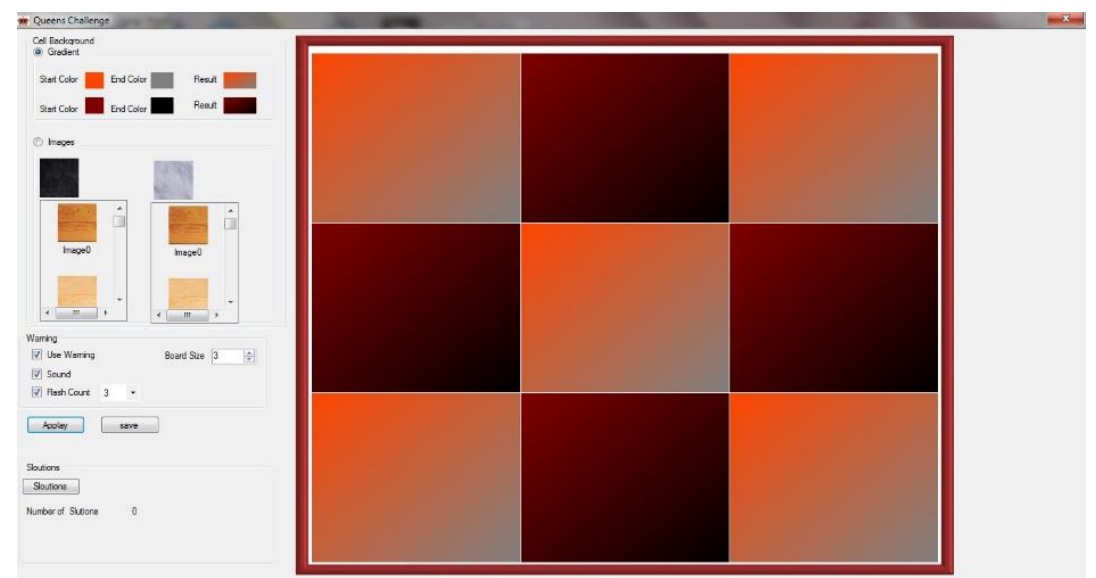

Figure 14. Game Activity- Choose Level 3

There are no solution in levels 2 and 3 because it is impossible to place 2 queens on $2 \times 2$ board or place 3 queens on $3 \times 3$ board without attack each other. Therefore, the number of solutions in these levels is zero. When press solutions button it, displays a dialog box stating no solution. Next screen captures depict that as shown in Figures 15 and 16 respectively. 


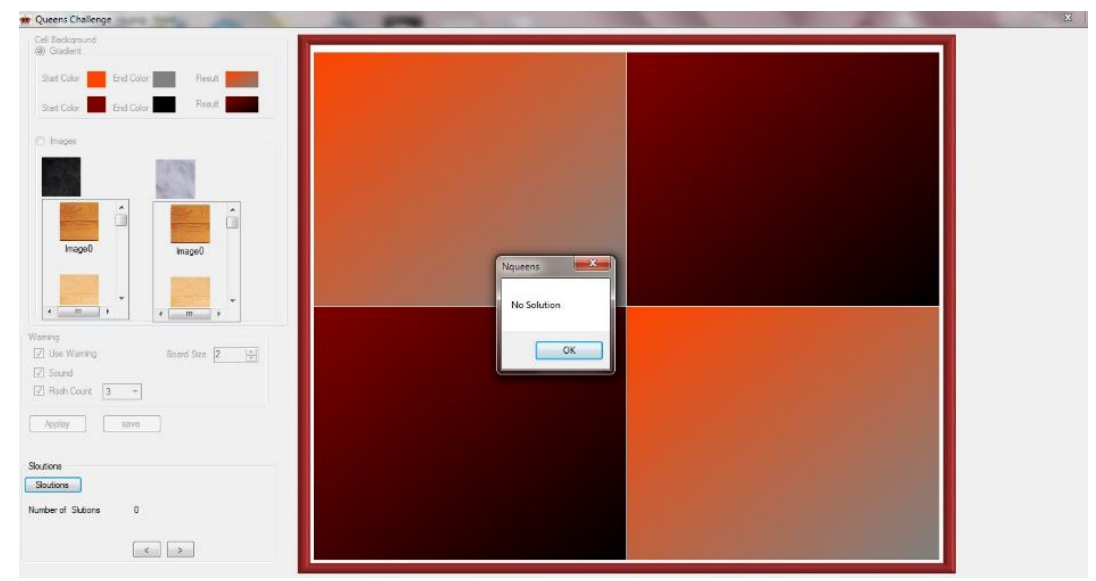

Figure 15. Game Activity- Solution Level 2

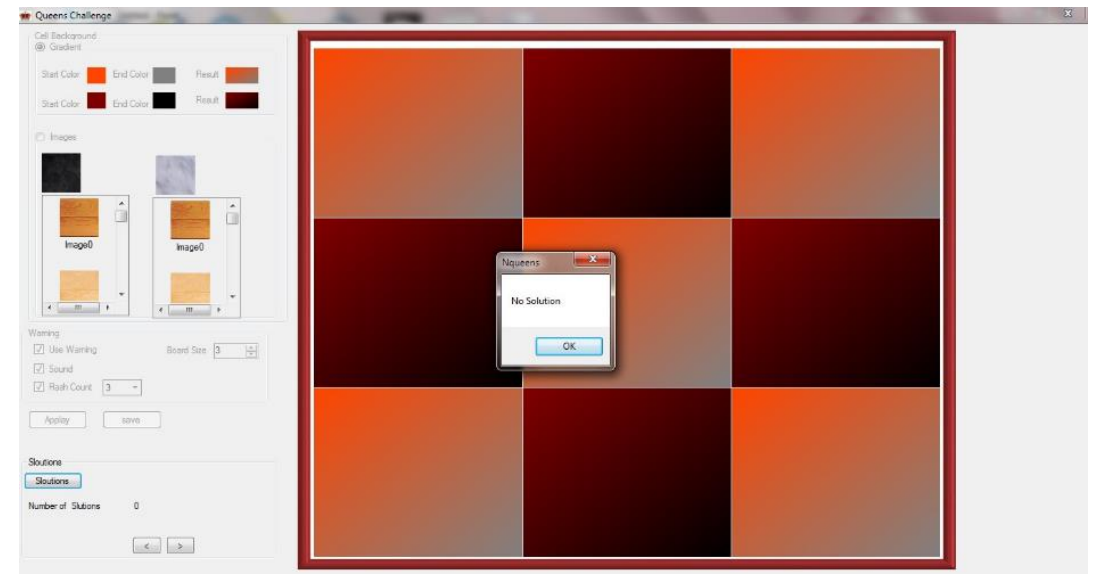

Figure 16. Game Activity- Solution Level 3

\section{- Sample Report}

We are to improve the system therefore, it can provide 1 to 25 game levels. The player can select game level by their own. Figure 17 Choose Level 1, Figure 18 Choose Level 3, Figure 19 Choose Level 8, Figure 20 Choose Level 15 and Figure 21 Choose Level 21 and Figure 22 Choose Level 25.

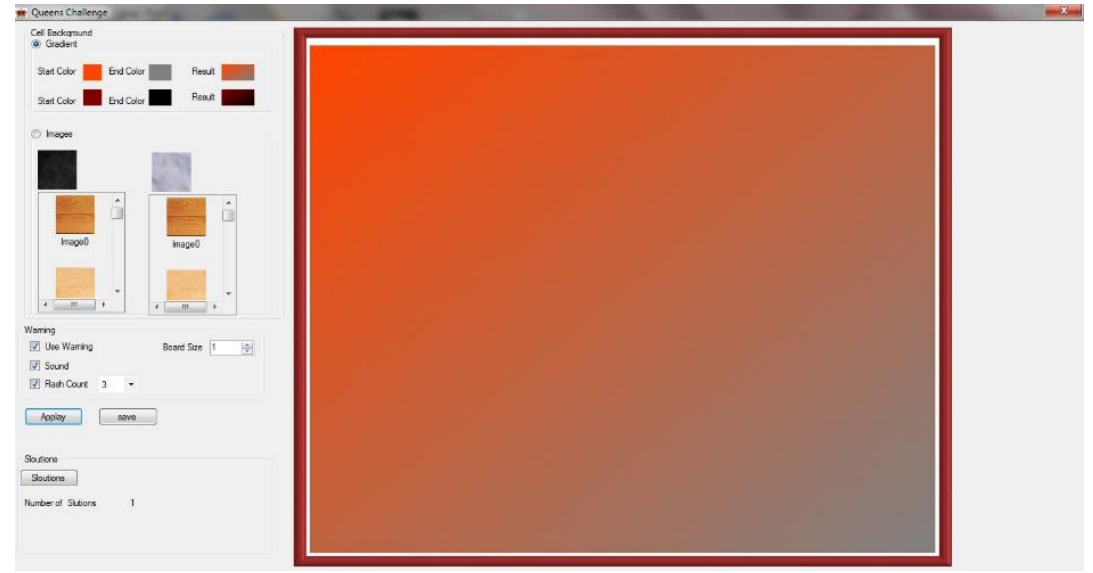

Figure 17. Choose Level 1 


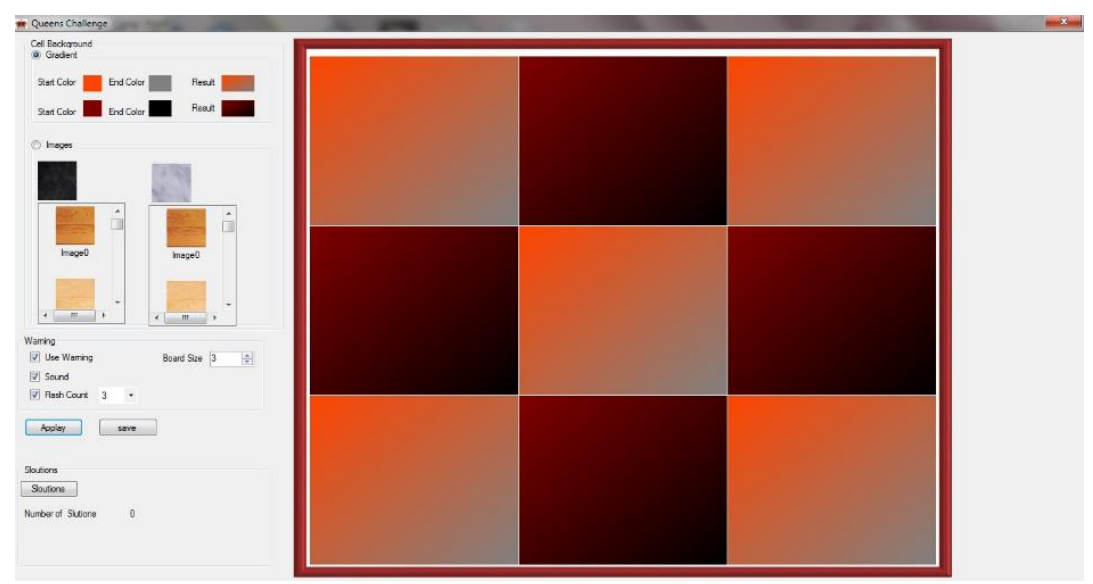

Figure 18. Choose Level 3

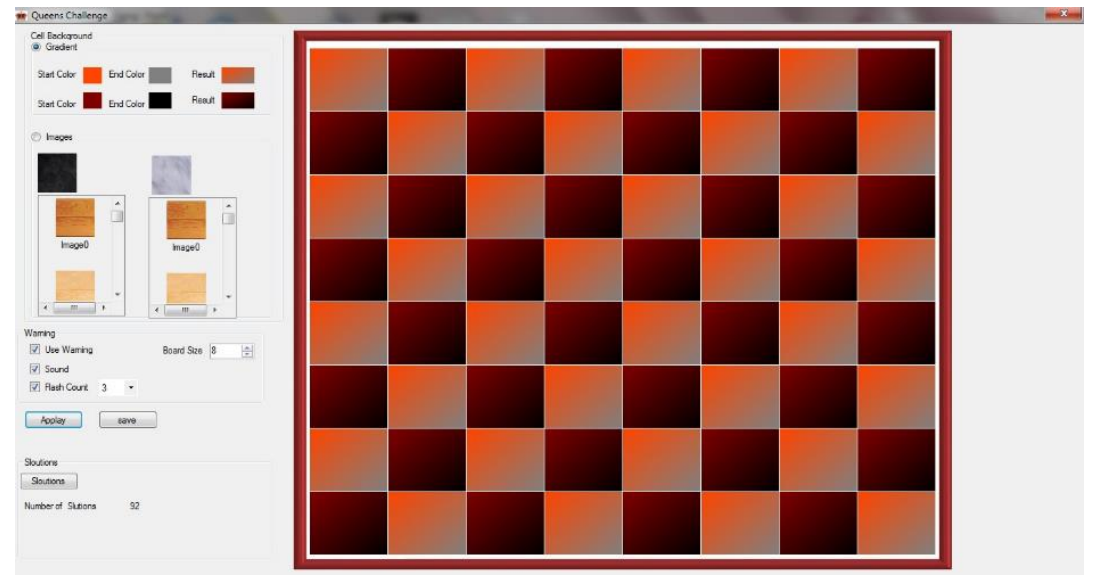

Figure 19. Choose Level 8

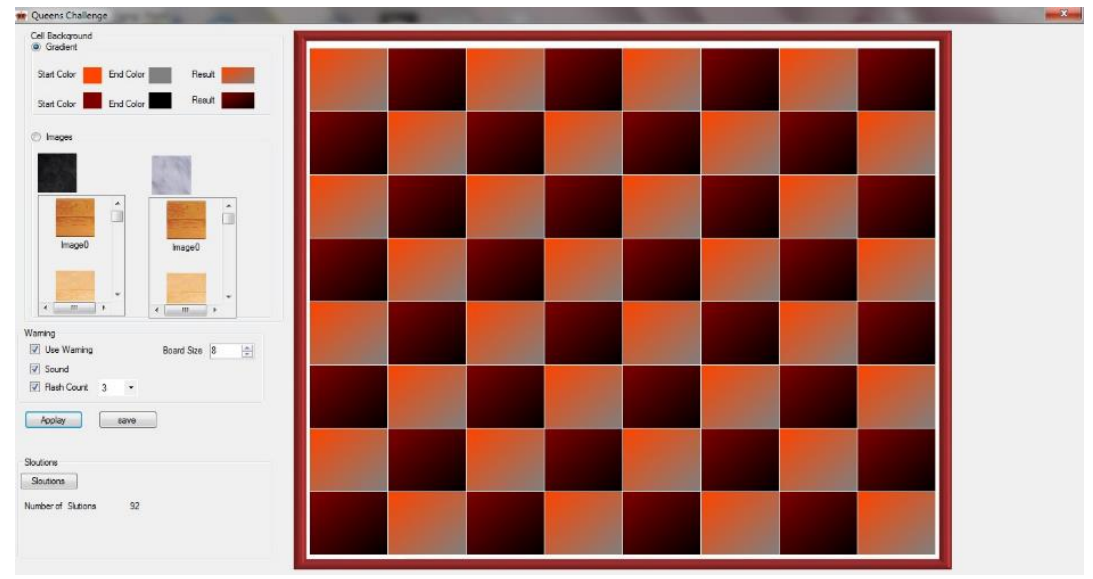

Figure 20. Choose Level 15 


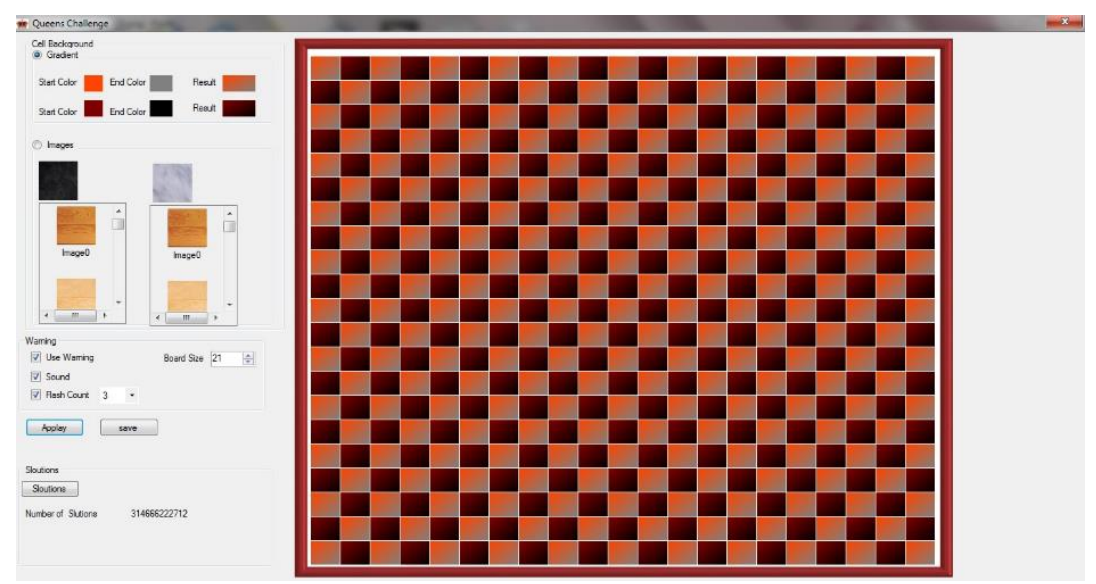

Figure 21. Choose Level 21

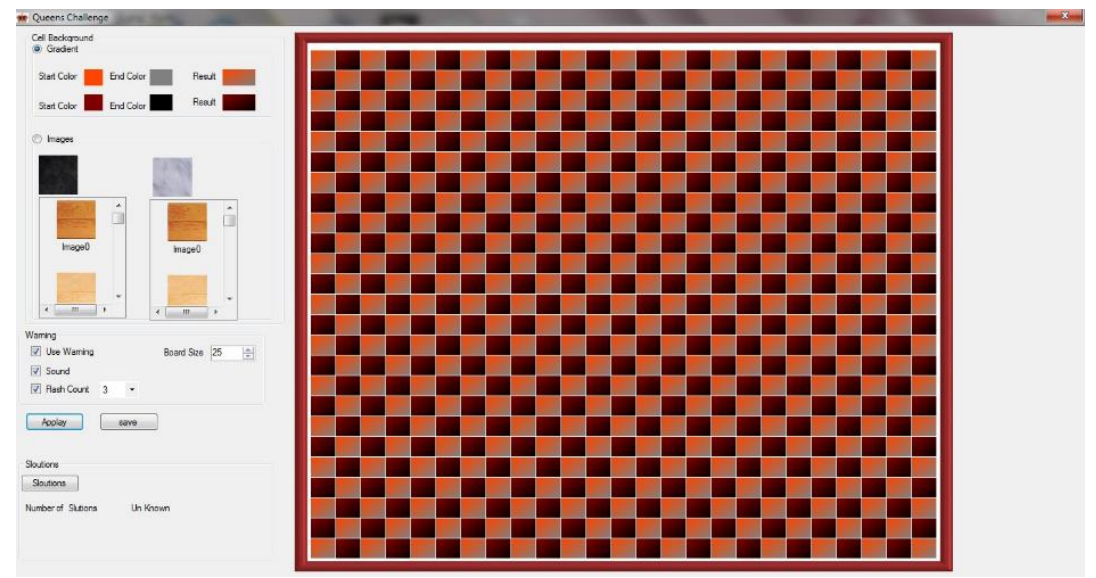

Figure 22. Choose Level 25

\section{- Pseudo Codes}

Public Class splash

Private Sub Timer1_Tick(sender As Object, e As EventArgs) Handles Timer1.Tick

MainQueen.Show()

MainQueen. WindowState $=$ FormWindowState. Maximized

Hide()

Timer1.Stop()

End Sub

Private Sub splash_Load(sender As Object, e As EventArgs) Handles MyBase.Load Timer1.Start() End Sub End Class

Figure 23. Splash Events 


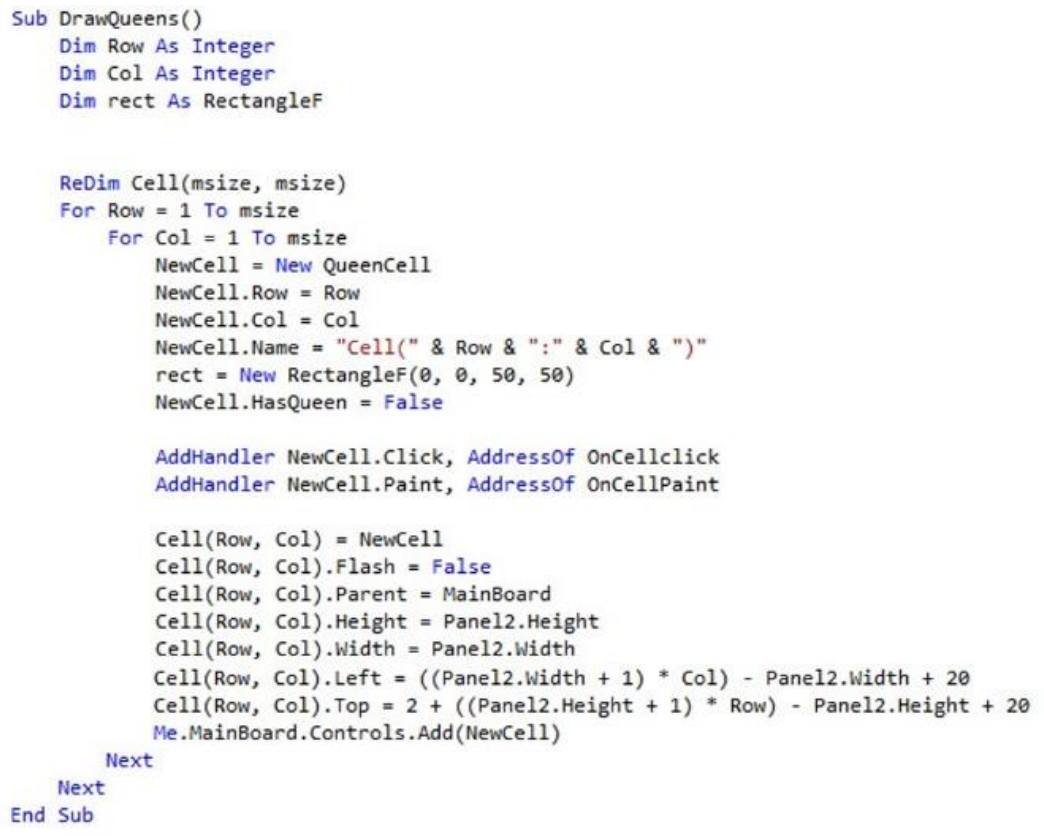

Figure 24. Draw Queens

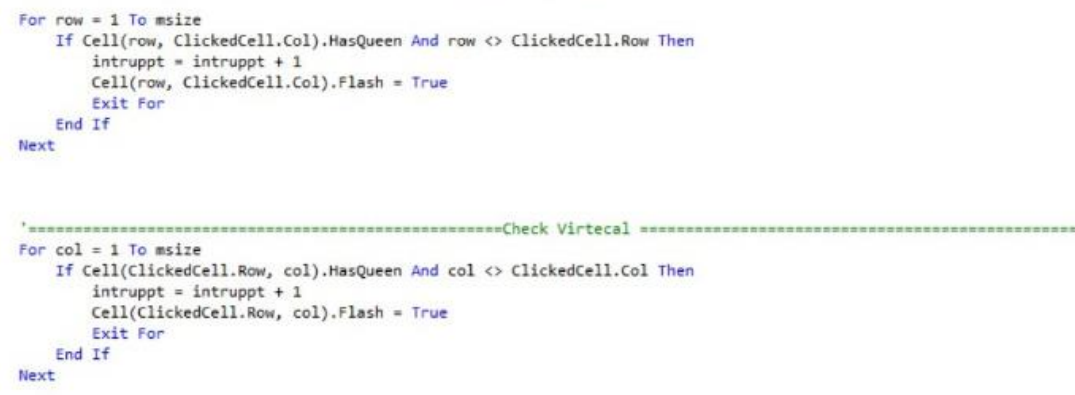

Figure 25. Check Horizontal and Vertical

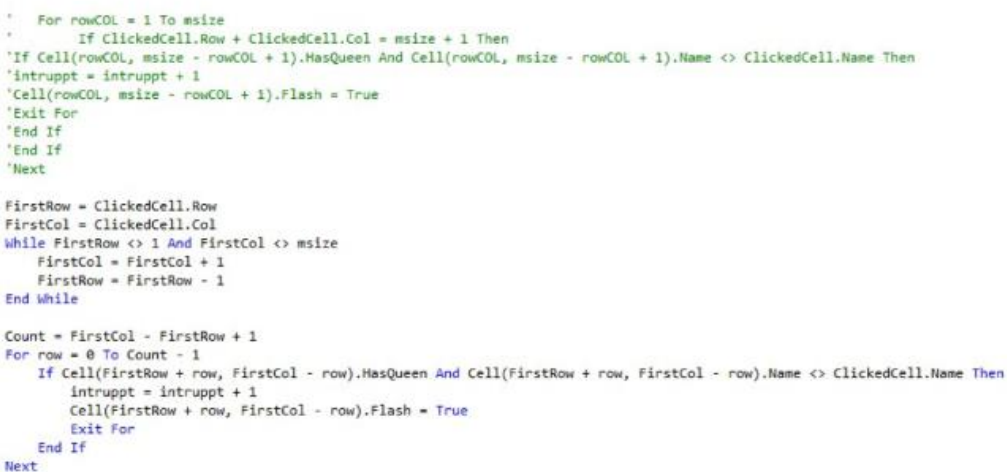

Figure 26. Check Diagonal। 


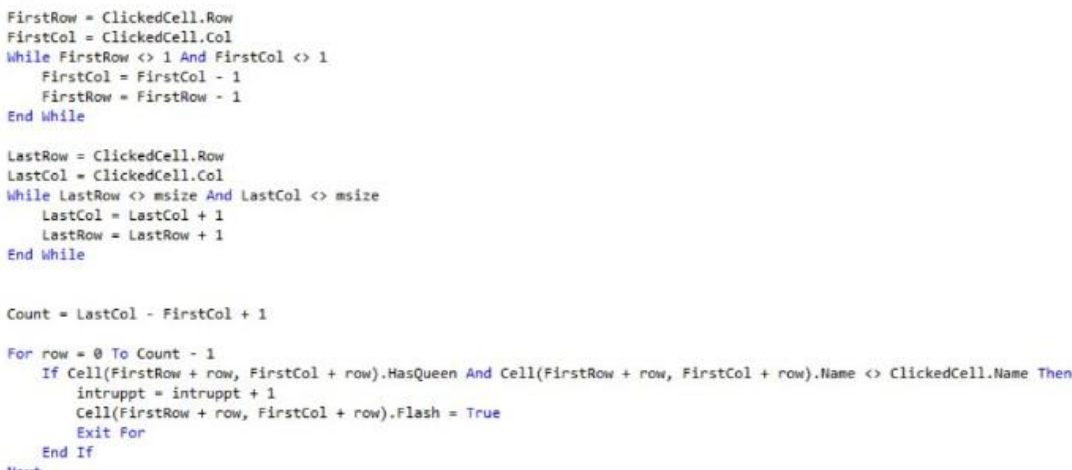

Figure 27. Check Diagonal /

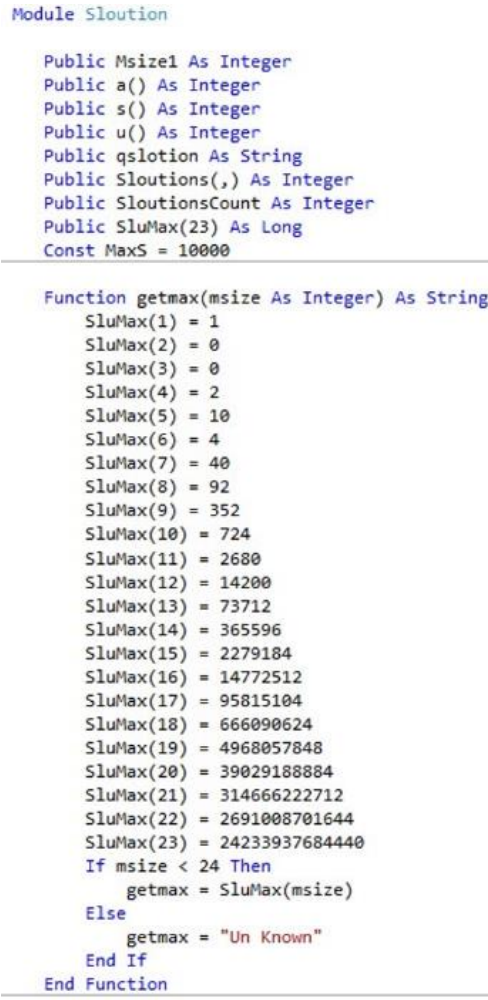

Figure 28. Number of Solutions 


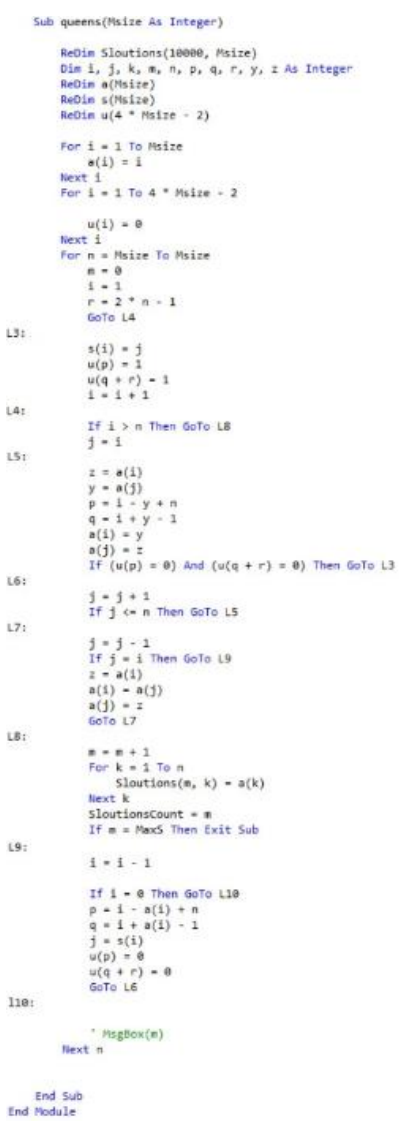

Figure 29. Solution Algorithm 


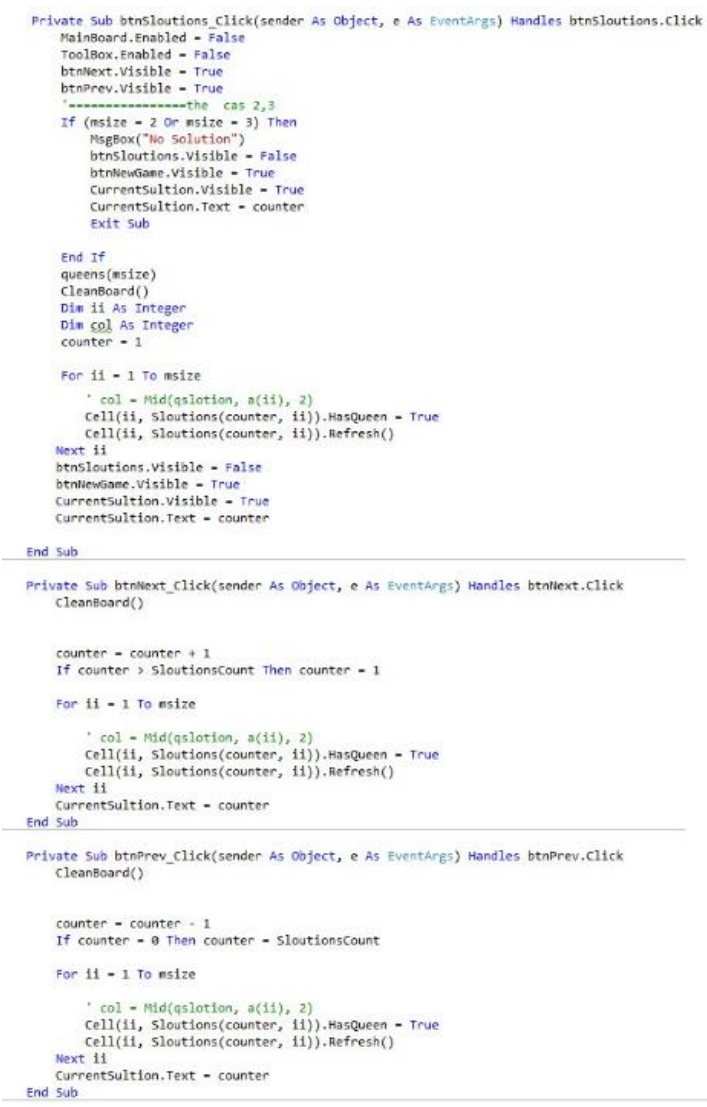

Figure 30. View Solutions

\section{Methodology and Hypotheses Development}

The evaluation of the proposed game aims to estimate the degree of its prosperity, assessing whether the intended interest group accomplished the characterized destinations. Evaluation should cover both the incorporate several levels from $1 \times 1$ until $25 \times 25$ of the proposed game, as well as the usability and usefulness of the game as shown in Figure 31 .

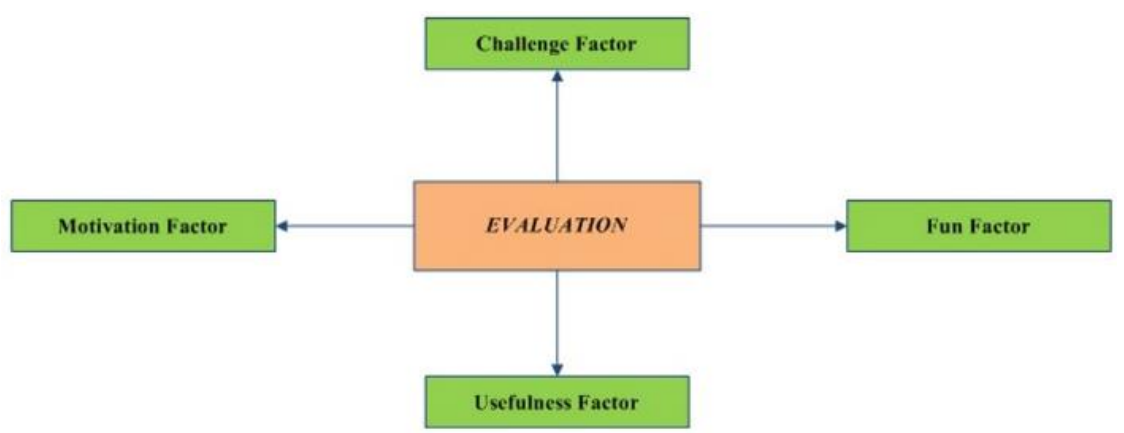

Figure 31. Evaluation Factors

The first factor is Challenge: The game should adequately test the player's competency level. The expansion of trouble ought to happen at a fitting pace going with the expectation to absorb information. New hindrances and circumstances ought to be introduced all through the game to limit weariness and to keep the players intrigued (Sweetser and Wyeth, 2005; Sweetser et al., 2012). It is to accomplish objectives, players should apply exertion, and the exertion is a challenge. Despite the fact that challenge is certifiably not an important factor for a game, the way toward arriving at objectives is ordinarily joined by difficulties (Johnson, 2012; Petri and Gresse, 2016). 
Demonstrated that games are ordinarily testing, regularly propel players, and enjoy them or discourage them when they accomplish objectives or come up short. A challenge regularly requires player capacities, for example, exactness, strong control, and brisk reasoning (Bourque and Fairley, 2014).This factor is related to game mechanisms and game fantasy. The second factor is Motivation: This means a feeling of relaxation, happiness, pleasure and diversion (Poels et al, 2007; Savi et al., 2011). The significance of a game's encounter relies upon how much general interest it can create. Making and keeping the player's interest is the best approach to deal with his motivation. His motivation is the factor that will decide whether a player will keep playing following a few minutes, just as to what extent he will play and whether he will complete the game. The third factor is Fun: That implies inspiration enables the player to have an encounter of profound contribution inside the game, making a test with certifiable center, then she/he disregards the outside world during interactivity (Fu et al., 2009). The forth factor is Usefulness: This implies that the proposed eight queens chess puzzle game is used by a specific age group to evaluate their knowledge, learning decision-making, and point of view. In addition to, save time, money and effort by collecting a series of episodes in a single game (Abu-Dalbouh, 2017; Abu-Dalbouh, 2016a ; Abu-Dalbouh, 2016b; Abu-Dalbouh, 2016c; Abu-Dalbouh et al., 2015a; Abu-Dalbouh et al., 2015b; Abu-Dalbouh, 2013).

In light of the above research, the hypotheses are proposed as below:

HYPOTHESIS 1: Incorporating several levels of the puzzle game in one program it make the game more exciting and challenging. (Challenge Factor).

HYPOTHESIS 2: Incorporating several levels of the puzzle game in one program will enhance the performance and motivation. (Motivation Factor).

HYPOTHESIS 3: There is a high impact and influence between incorporating several levels in one program and increase playing the game (Fun Factor).

HYPOTHESIS 4: Incorporating several levels of the puzzle game in one program, it will save time, money and effort by collecting all levels in a single game with series of episodes. The use of the available literature reviews (Sureshchandar et al., 2002; Thomas et al., 2002; Yang et al., 2004; Ellis et al., 2007) was the major refinement of the criteria for the proposed game. An empiric examination, depending on the questionnaire, has been tested. For the information assortment, the structured questionnaire was used to investigate the four dimensions. The questionnaire was designed based on the proposed evaluation namely; Challenge, Fun, Motivation and Usefulness factors. The questionnaire consists of four dimensions.

The first dimension to examine the Hypotheses 1: (Incorporating several levels of the puzzle game in one program it make the game more exciting and challenging).

The second dimension to examine the Hypotheses 2: (Incorporating several levels of the puzzle game in one program will enhance the performance and motivation).

The third dimension to examine the Hypotheses 3: (There is a high impact and influence between incorporating several levels in one program and increase playing the game).

The Fourth dimension to examine the Hypotheses 4: (Incorporating several levels of the puzzle game in one program, it will save time, money and effort by collecting all levels in a single game with series of episodes). As shown in Figure 32. 


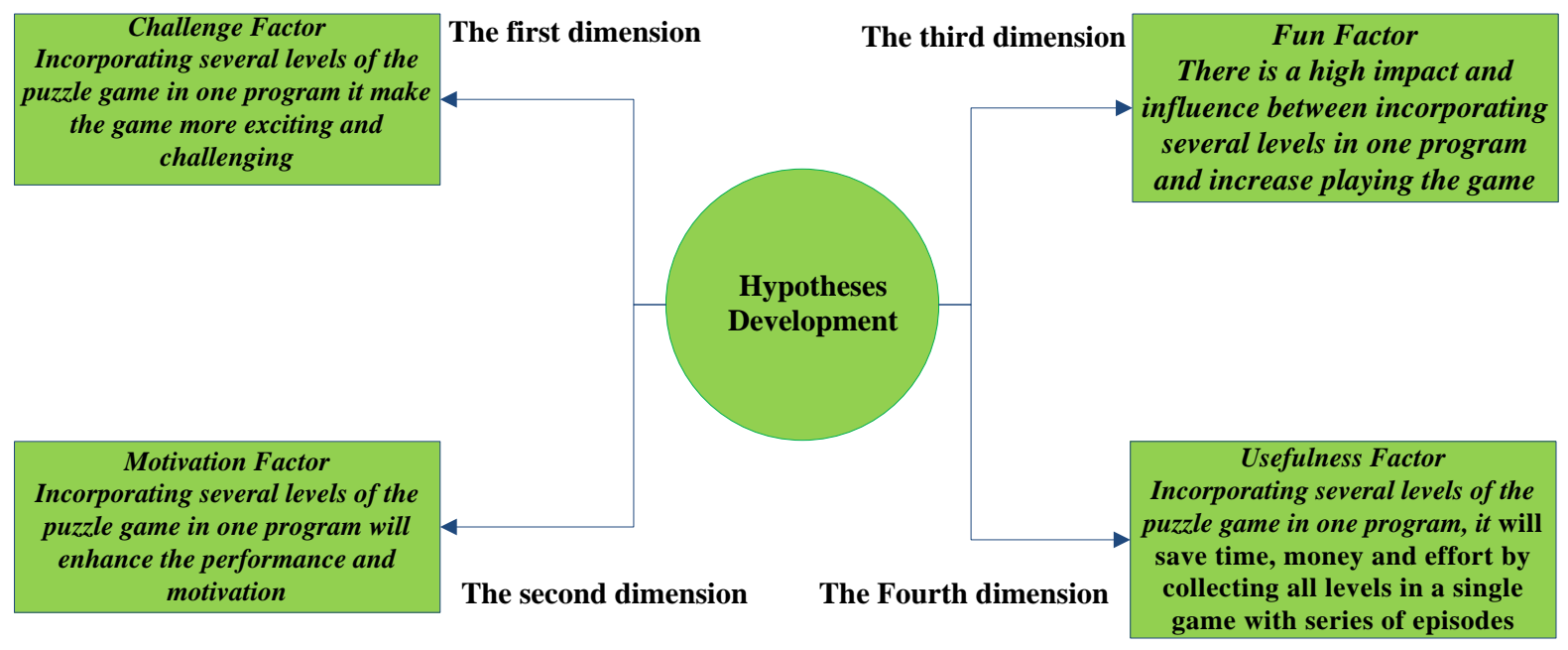

Figure 32. Hypotheses Development

\section{Results and Discussions}

The most important aspects of gaming assessment are the development of good criteria to achieve a reliable and legitimate standard from the referenced structure. The proposed game has been validated by a questionnaire-based empirical study. Descriptive statistics on the questions revealed that the players had achieved the goals of the game by applying their knowledge and that the players had positive feelings about the effectiveness of the proposed game. In addition, the players have shown that the controls to perform the actions in the proposed game have responded well. The results demonstrated that the players in the sample enjoy themselves by playing the proposed game. The implemented game enhances the standard view of enjoyment by taking into account the adversarial tensions embodied in both the sentiments of challenge and concentration, as most of the responses to these features were more than midpoints of their particular dimensions.

Queens challenge is a game that can solve queens puzzles. The user will be able to view the solution. In either case, the software is going to be able to solve the puzzle completely. At any time, the user will be able to know whether to put the queen in the wrong place. It shows, therefore, that the sample players have fun with the game and like to play this game again. In addition, the queen challenge Game players will feel interesting and happy to play this game. Several levels are included, along with the proposed 8-queen puzzle game, which will save time, effort and money by downloading each level separately. Queen challenge game is user-friendly and has clear instructions and a friendly interface. Table 1 shows the respondents' agreements about the dimensions and Figure 33 shows the respondents' agreements for all items. 


\begin{tabular}{|c|c|}
\hline $\begin{array}{c}\text { Item 1: I achieved the goals of the game applying my } \\
\text { knowledge }\end{array}$ & $81.4 \%$ \\
\hline $\begin{array}{l}\text { Item 2: I had positive feelings on the efficiency of this } \\
\text { game }\end{array}$ & $76.4 \%$ \\
\hline $\begin{array}{c}\text { Item 3: The controls to perform actions in the game } \\
\text { responded well }\end{array}$ & $63.9 \%$ \\
\hline $\begin{array}{c}\text { Item 4: It's easy to learn how to use the interface and } \\
\text { game controls }\end{array}$ & $84.5 \%$ \\
\hline
\end{tabular}

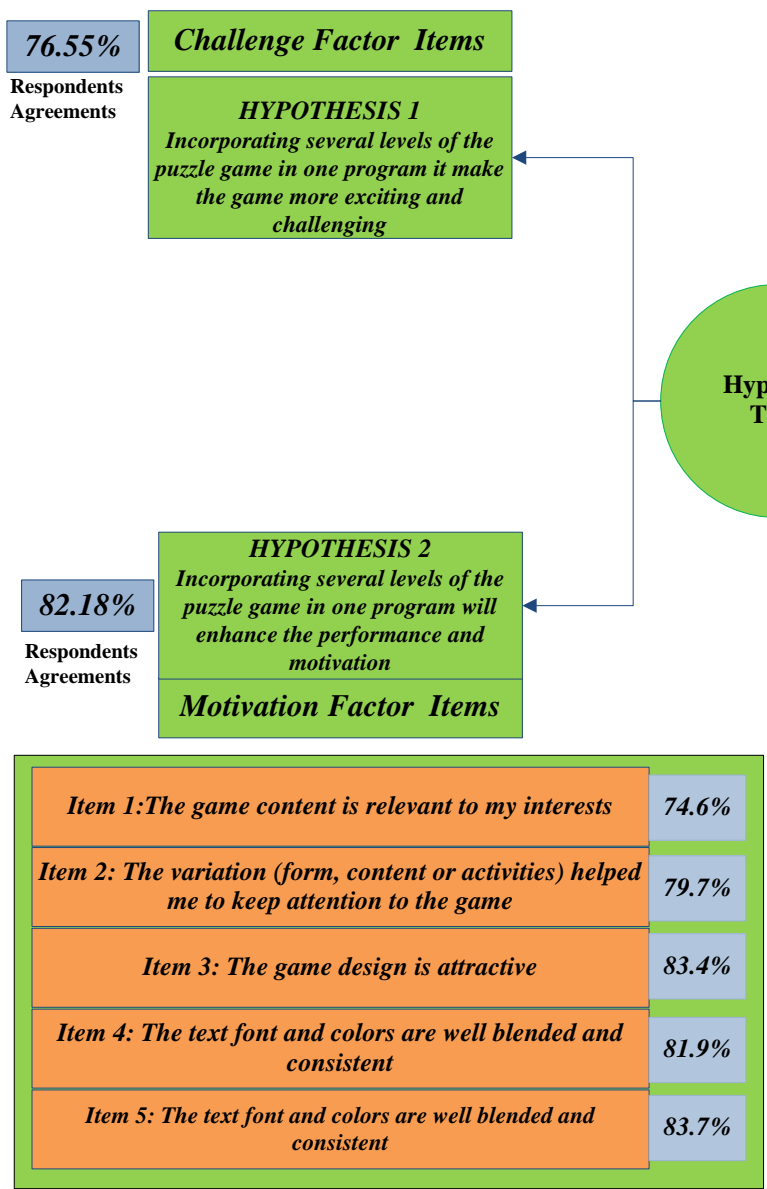
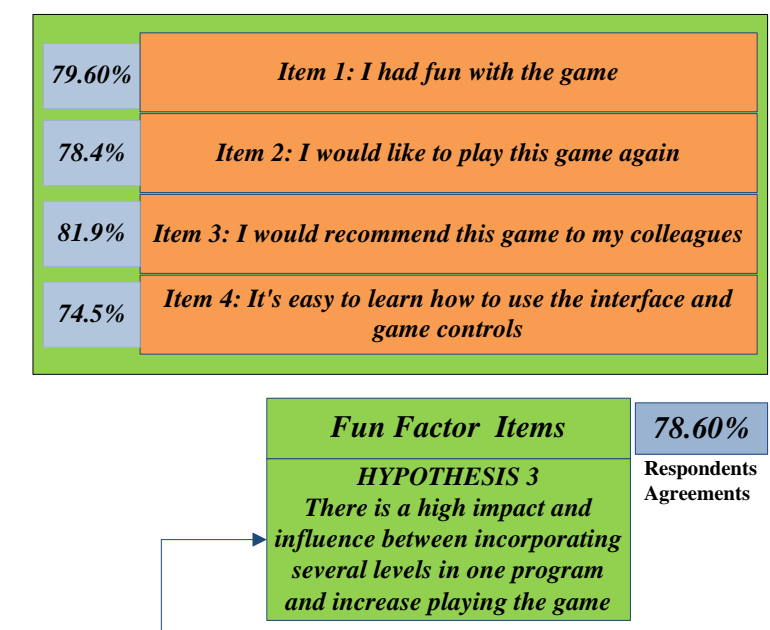

\section{HYPOTHESIS 4}

Incorporating several levels of the puzzle game in one program, it will save time, money and effort by collecting all levels in a single game with series of episodes Agreements

\begin{tabular}{|c|c|}
\hline $83.4 \%$ & $\begin{array}{c}\text { Item 1: Eight queens chess puzzle game system } \\
\text { enhances creativity }\end{array}$ \\
\cline { 2 - 2 } $81.4 \%$ & $\begin{array}{c}\text { Item 2: Eight queens chess puzzle game system involves } \\
\text { problem solving techniques }\end{array}$ \\
\hline \multirow{2}{*}{$78 \%$} & $\begin{array}{c}\text { Item 3: Eight queens chess puzzle game system will } \\
\text { improve decision-making and thinking strategies }\end{array}$ \\
\hline \multirow{2}{*}{$82 \%$} & $\begin{array}{c}\text { Item 4: Eight queens chess puzzle game system allows } \\
\text { players to test their consistency, skills and strategy while } \\
\text { competing in a more levels }\end{array}$ \\
\cline { 2 - 2 } $89.4 \%$ & $\begin{array}{c}\text { Item 5: Eight queens chess puzzle game system is added } \\
\text { more enjoyment and excitement }\end{array}$ \\
\hline \multirow{2}{*}{$66 \%$} & $\begin{array}{c}\text { Item } 6: \text { Eight queens chess puzzle game system will help } \\
\text { the players by displaying all solutions while playing }\end{array}$ \\
\hline \multirow{2}{*}{$3.9 \%$} & $\begin{array}{c}\text { Item } 7: \text { Eight queens chess puzzle game system will save } \\
\text { time, effort and money from downloading each level } \\
\text { separately }\end{array}$ \\
\hline
\end{tabular}

Figure 33. Respondents agreements

Table 1. Game Factors Questionnaire

\section{Dimensions}

Respondents

Agreements

\section{First Dimension: Challenge factor Items}

I achieved he goals of the game applying my know ledge

I had positive feelings on efficiency of this game

The controls to perform action in the game responded well

It is easy to learn how to use the interface and game controls

\section{Second Dimension: Motivation Factor Items}


The game content is relevant to my interests

The variation (form, content or activities) helped me to keep attention to the game

The game design is attractive

The text font and colors are well blended and consistent

Incorporating several levels from $1 \times 1$ until $25 \times 25$ of the puzzle game in one program encourage me to play more

\section{Third Dimension: Fun Factor Items}

I had fun with the game

I would like to play this game again

I would recommend this game to my colleagues

I was exiting to play next level

\section{Fourth Dimension: Usefulness Factor Items}

Eight queens chess puzzle game system enhances creativity

Eight queens chess puzzle game system involves problem solving techniques

Eight queens chess puzzle game system will improve decision making and thinking strategies

Eight queens chess puzzle game system allows players to test their consistency, skills and strategy while competing in a more levels

Eight queens chess puzzle game system is added more enjoyment and excitement

Eight queens chess puzzle game system will help the players by displaying all solutions while playing

Eight queens chess puzzle game system will save time, effort and money from downloading each level separately

\section{Conclusion and Future work}

For decades, artificial intelligence researchers aimed to build game-playing systems configured to organize brains with the most focused people in the world. The achievement of such methodologies have a significant result of improved playing computations and extended periods of information-designing exercise in the interest of program engineers, physically adding game-specific information to their systems. The proposed game has designed, implemented and tested with users successfully. The study helped in understanding the challenges. It also helped in understanding the value of designing the components of overall application before implementing them. The prototype has many stages, from 1 to 1 to 25 to 25 , but it is possible to find each stage in a separate game. This will cause the players a lot of trouble and each stage should be downloaded to finish the challenge. This will lead to spend more time and efforts from the players and the cost for every level will cost the players more and more either.

Therefore, the aim of this article is to integrate multiple levels of the game instead download each level separately to save time, effort and money. Then develops the proposed prototype and demonstrates all the solutions when playing the puzzle game at any level. In this study, we are collected all levels in a single game with series of episodes, and it will have advantages regarding the total cost, effort, time, and money. In addition, the proposed game added additional levels to 25 , to make the game more exciting and challenging even for the brilliant minds. Moreover, this game is used by a specific age group to test their intellect, judgment ability, and way of doing things. A player starts gradually from the easiest level to the hardest one, and the game provides all the possible solutions for all levels.

\section{Acknowledgments}

Researchers would like to thank the Deanship of Scientific Research, Qassim University for funding publication of this project. 


\section{References}

Abu-Dalbouh, H. (2016a). An Integrated Expert User with End User in Technology Acceptance Model for Actual Evaluation. Computer and Information Science - Canadian center of science and education, 9(1), 47-53. https://doi.org/10.5539/cis.v9n1p47

Abu-Dalbouh, H. (2016b). Implementing End-User Privacy Through Human Computer Interaction for Improving Quality of Personalized Web. Computer and Information Science - Canadian center of science and education, 9(1), 75-89. https://doi.org/10.5539/cis.v9n1p75

Abu-Dalbouh, H. (2016c). Using a Modified Technology Acceptance Model to Evaluate Designing Eight Queens Chess Puzzle Game. Journal of Computer Science. J. Comput. Sci., 12(5), 232-240. June 2016. https://doi.org/10.3844/jcssp.2016.232.240

Abu-Dalbouh, H. M. (2013). A Questionnaire Approach Based on the Technology Acceptance Model for Mobile Tracking on Patient Progress Applications. J. Comput. Sci., 9(6), 763-770. ttps://doi.org/10.3844/jcssp.2013.763.770

Abu-Dalbouh, H., Al-Buhairy, M., \& Al-Motiry, I. (2017). Applied the Technology Acceptance Model in Designing a Questionnaire for Mobile Reminder System. Computer and Information Science - Canadian center of science and education, 10(2), 15-24. https://doi.org/10.5539/cis.v10n2p15

Abu-Dalbouh, H., Al-Habeeb, A., Al-Kholifi, A., Al-Motiry, I., \& Al-Buhairy, M. (2015b). A Mobile Reminder System for Elderly and Alzheimer's Patients. International Journal of Computer Science Issues. International Journal of Computer Science Issues (IJCSI), 12(5), 95-101. Retrieved from http://ijcsi.org/papers/IJCSI-12-5-95-101.pdf

Abu-Dalbouh, H., Al-Roieshdie, E., Al-Otaibi, A., Al-Ma7ana, B., Al-Mutairi, H., Al-Mutairi, M., \& Al-Harbi, N. (2015a). A Proposed Website to Evaluate the Academic Performance in College of Sciences and Arts in Unaizah. Research Journal of Applied Sciences, Engineering and Technology, 11(12), 1305-1319. https://doi.org/10.19026/rjaset.11.2239

AL-Obaidi, B., Ali, S., \& Jassim, D. (2020). Influence of a river water quality on the efficiency of water treatment using artificial neural network. Journal of Engineering Science and Technology, 15(4), 2610-2623.

Arnoldy, B. (2006). Sudoku Strategies. The Home Forum (The Christian Science Monitor), Schaschek, Sarah, March 2006.

Bikku, T., \& Sree, S. (2020). Deep learning approaches for classifying data: a review. Journal of Engineering Science and Technology, 15(4), 2580-2594.

Bourque, P., \& Fairley, R. E. (2014). Swebok v3.0 Guide to the software enginnering body of knowledge. IEEE Computer Society, 2014.

Boyer, C. (2009). Sudoku's French ancestors. Retrieved August 3, 2009, from http://web.archive.org

Dina, N. Z., Sabilla, W. I., \& Kartono. (2019). The impact of using visual learning environment on student programming course learning achievement: a case study of universitas airlangga. (2019). Journal of Engineering Science and Technology, 14(2), 712-725.

Ellis, H., Sartaj, S., \& Sanguthevar, R. (2007). Fundamental of Computer algorithms. Galgotia Publications Pvt. Ltd.

Fu, F., Su, R., \& Yu, S. (2009). E Game Flow: A scale to measure learners' enjoyment of elearning games. Computers \& Education, 52(1), 101-112. https://doi.org/10.1016/j.compedu.2008.07.004

Hoffer, J. A. (2011). Modern System Analysis and Design (6ed.). Prentice Hall.

Johan, H., \& Homo, L. (1955). A Study of the Play Element in Culture. Boston: Beacon Press.

Johnson, M. (2012). A pilot study examining the motivational effect of instructional materials on EFL learning motivation. Journal of Language and Culture of Hokkaido, 10, 39-47.

Petri, G., Gresse, C., Wangenheim, V., \& Borgatto, A. F. (2016). MEEGA+: an evolution of a model for the evaluation of educational games. Technical Report, INCoD/GQS.03.2016.E., 2016. https://doi.org/10.1007/978-3-319-08234-9_214-1

Poels, K., Kort, Y. D., \& Ijsselsteijn, W. (2007). It is always a lot of fun!: exploring imensions of digital game 
experience using focus group methodology. Proc. of Conf. on Future Play, pp. 83-89, Toronto, Canada, 2007. https://doi.org/10.1145/1328202.1328218

Savi, R., Gresse, C., Wangenheim, V., \& Borgatto, A. F. (2011). A model for the evaluation of educational games for teaching software engineering. Proc. of the 25th Brazilian Symposium on Software Engineering, 2011, pp. 194-203. São Paulo, Brazil (in Portuguese). https://doi.org/10.1109/SBES.2011.27

Sureshchandar, G. S., Rajendran, C., \& Anantharaman, R. N. (2002). The relationship between service quality and customer satisfaction-a factor specific approach. Journal of services marketing, 16(4), 363-379. https://doi.org/10.1108/08876040210433248

Sweetser, P., \& Wyeth, P. (2005). GameFlow: a model for evaluating player enjoyment in games. Computers in Entertainment, 3(3), 1-24. https://doi.org/10.1145/1077246.1077253

Sweetser, P., Johnson, D., Wyeth, P., \& Ozdowska, A. (2012). Game Flow heuristics for designing and evaluating real-time strategy games. Proc. of the 8th Australasian Conf. on Interactive Entertainment: Playing the System, ACM, New York, NY, USA, Article 1, 10 pages, 2012. https://doi.org/10.1145/2336727.2336728

Thomas, H. C., Charles, E. L., Ronald, L. R., \& Clifford, S. (2002). Introduction to Algorithms (2nd ed.). PHI.

Yang, Z., Jun, M., \& Peterson, R. T. (2004). Measuring customer perceived online service quality: scale development and managerial implications. International Journal of Operations \& Production Management, 24(11), 1149-1174. https://doi.org/10.1108/01443570410563278

\section{Copyrights}

Copyright for this article is retained by the author(s), with first publication rights granted to the journal.

This is an open-access article distributed under the terms and conditions of the Creative Commons Attribution license (http://creativecommons.org/licenses/by/4.0/). 